\title{
Investigation of Correlated Rayleigh Fading Channel with Alamouti's STBC-MRC system
}

\author{
Tanvir Ahmed, Md. Shamim Anower, Md. Zahurul Islam Sarkar and Md. Mortuza \\ Ali \\ Rajshahi University of Engineering and Technology \\ tavnir_eee_ruet@yahoo.com
}

\begin{abstract}
The effect of antenna diversity for a double transmit and multiple receive antenna supported wireless communication system is investigated, that employs multi user Alamouti's space time block coding (STBC) and maximal ratio combining (MRC) scheme on secured text message transmission. The FEC encoded Alamouti's-MRC transmission system under investigation implements RSA cryptographic algorithm and deploys various multi-level digital modulations (16-PSK and 16-QAM) techniques over an Additive White Gaussian Noise (AWGN) and Correlated Rayleigh Fading Channels. Rayleigh channel correlation parameter $\rho$ is varied from 0 to 0.9 . It has been observed from the study that in case of without receive antenna diversity the system shows comparatively worst performance in 16PSK scheme and satisfactory performance in 16-QAM for the correlation parameter $\rho=0.9$. It can also be seen that the system performance is improved with increase in number of receive antenna. The performance analysis shows that with implemented Alamouti's-MRC scheme under 16-QAM digital modulation, the system provides excellent performance over a significantly low signal to noise ratio (SNR) values.
\end{abstract}

Keywords: MRC, Correlated Rayleigh Fading, STBC, RSA

\section{Introduction}

Multiple-input multiple-output (MIMO) technology has rapidly gained in popularity over the past decade due to its powerful performance-enhancing capabilities. Communication in wireless channels is impaired predominantly by multi-path fading. MIMO technology constitutes a breakthrough in wireless communication system design. The technology offers a number of benefits that help to meet the challenges posed by both the impairments in the wireless channel as well as resource constraints [1]. It is well known that correlation in fading across multiple diversity channels results in a degradation of the diversity gain obtained [2]. Ergodic capacity of maximal ratio combining (MRC) schemes over arbitrarily correlated Rician fading channels is obtained in [2] and find out the effects of channel correlation on the ergodic capacity. It shows that both the phase and the magnitude of correlation have an impact on the ergodic capacity of Rician fading channels. When channel knowledge is available at both the transmitter and receiver, MIMO transmit beamforming with maximum ratio combining (MRC) receivers [3, 4] is particularly robust against the severe effects of fading. Recently, MIMO-MRC has been investigated in uncorrelated and semicorrelated channel scenarios [5]. In [5] it is proved that MIMO-MRC achieves the maximum available spatial diversity order, and demonstrated the effect of spatial correlation. The ergodic capacity of MRC with arbitrarily correlated Rician faded branches is computed and the effects of correlated branches on the performance of MRC diversity are determined in [6]. In [7-11], 
uncorrelated Rayleigh fading was considered, and the output SNR statistical properties were derived based on maximum eigen value statistics of complex central Wishart matrices. In [12] performance analysis of maximal ratio combining (MRC) using BPSK modulation in spatially correlated Rayleigh fading channels with imperfect channel knowledge are presented in terms of antenna array configuration and parameters of scatterer distributions. [13] shows performance analysis of maximal ratio combining (MRC), selection combining (SC), and equal gain combining (EGC) diversity reception with two correlated Rayleigh-fading signals.

Thus far, to the best of the author's knowledge, there is no such work available about the performance analysis presented here. We shall consider the case of the simple Alamouti's space-time block code as it is the only scheme which can provide full rate and full diversity for any signal constellations. Network safety measures are provided using RSA algorithm to shield data during their communication in the proposed scheme. Therefore, this paper focuses on the evaluation of the BER performance for the FEC encoded Alamouti-MRC transmission system for various multi-level digital modulations techniques over an Additive White Gaussian Noise (AWGN) and correlated Rayleigh Fading Channel with the transmit diversity technique in conjunction with receive antenna diversity.

The paper is organized as follows. Section 2 presents the system description, which includes the transmitter, channel, and coherent receiver models. Performance analysis is presented in Section 3. Section 4 deals with the channel model. Finally, conclusions are drawn in Section 5.

\section{The Communication System Model}

The MIMO wireless communication system under consideration is shown in Figure 1. In such a communication system, four users are simultaneously transmitting their secured text messages. For secret message transmission the most widely used public-key cryptosystem RSA is used here. After encryption of plaintext of each user the ciphertext is converted into binary messages. The transmitted bits of each individual user are channel encoded by a convolutional encoder of rate $r=1 / 2$, interleaved for minimization of burst errors and then converted to M-ary signal. This M-ary signal is modulated using various types of multi level digital modulation techniques such as quadrature amplitude modulation (QAM), phase shift keying (PSK) and differentially phase shift keying (DPSK). The modulated digital signals are fed into the Alamouti Space Time Block Encoder, where the input stream is first segmented into two-symbol blocks. Each two-symbol block includes the first and second symbols $x_{1}$ and $x_{2}$, respectively. During the first symbol period, the encoder will send $x_{1}$ and $x_{2}$ to the first and second transmit antennas, respectively. During the next symbol period, $x^{*}{ }_{1}$ and $-x^{*}{ }_{2}$, where $*$ denotes complex conjugate will be sent to the first and second transmit antennas respectively. These two transmit antennas can either be collocated or distributed remotely if proper symbol timing synchronization scheme is adopted. Now the space-time encoded streams are sent to the wireless channels through the two transmit antennas. Assuming $r_{1}^{j}$ and $r_{2}^{j}$ are the received signals at the $j^{\text {th }}$ receive antenna at time $t$ and $t+T$, respectively, then $r_{1}^{j}$ and $r_{2}^{j}$ are given by [14-16] as follows.

$$
\begin{gathered}
r_{1}^{j}=h_{j 1} x_{1}+h_{j 2} x_{2}+n_{1}^{j} \\
r_{2}^{j}=-h_{j 1} x_{2}^{*}+h_{j 2} x_{1}^{*}+n_{2}^{j}
\end{gathered}
$$

where $h_{j i}, i=1,2 ; j=1,2, \ldots \ldots \mathrm{N}_{\mathrm{R}}$, is the fading coefficient for the path from transmit antenna $i$ to receive antenna $j$, and $n_{1}^{j}$ and $n_{2}^{j}$ are the noise signals for receive antenna $j$ at time $t$ and $t+$ 
$T$ respectively. The receiver constructs two decision statistics based on the linear combination of the received signals. The decision statistics are given by

$$
\begin{gathered}
\tilde{x}_{1}=\sum_{i=1}^{2} \sum_{j=1}^{N_{R}}\left|h_{j i}\right|^{2} x_{1}+\sum_{j=1}^{N_{R}} h_{j 1}{ }^{*} n_{1}^{j}+h_{j 2}\left(n_{2}^{j}\right)^{*} \\
\tilde{x}_{2}=\sum_{i=1}^{2} \sum_{j=1}^{N_{R}}\left|h_{j i}\right|^{2} x_{2}+\sum_{j=1}^{N_{R}} h_{j 2}{ }^{*} n_{1}^{j}-h_{j 1}\left(n_{2}^{j}\right)^{*}
\end{gathered}
$$

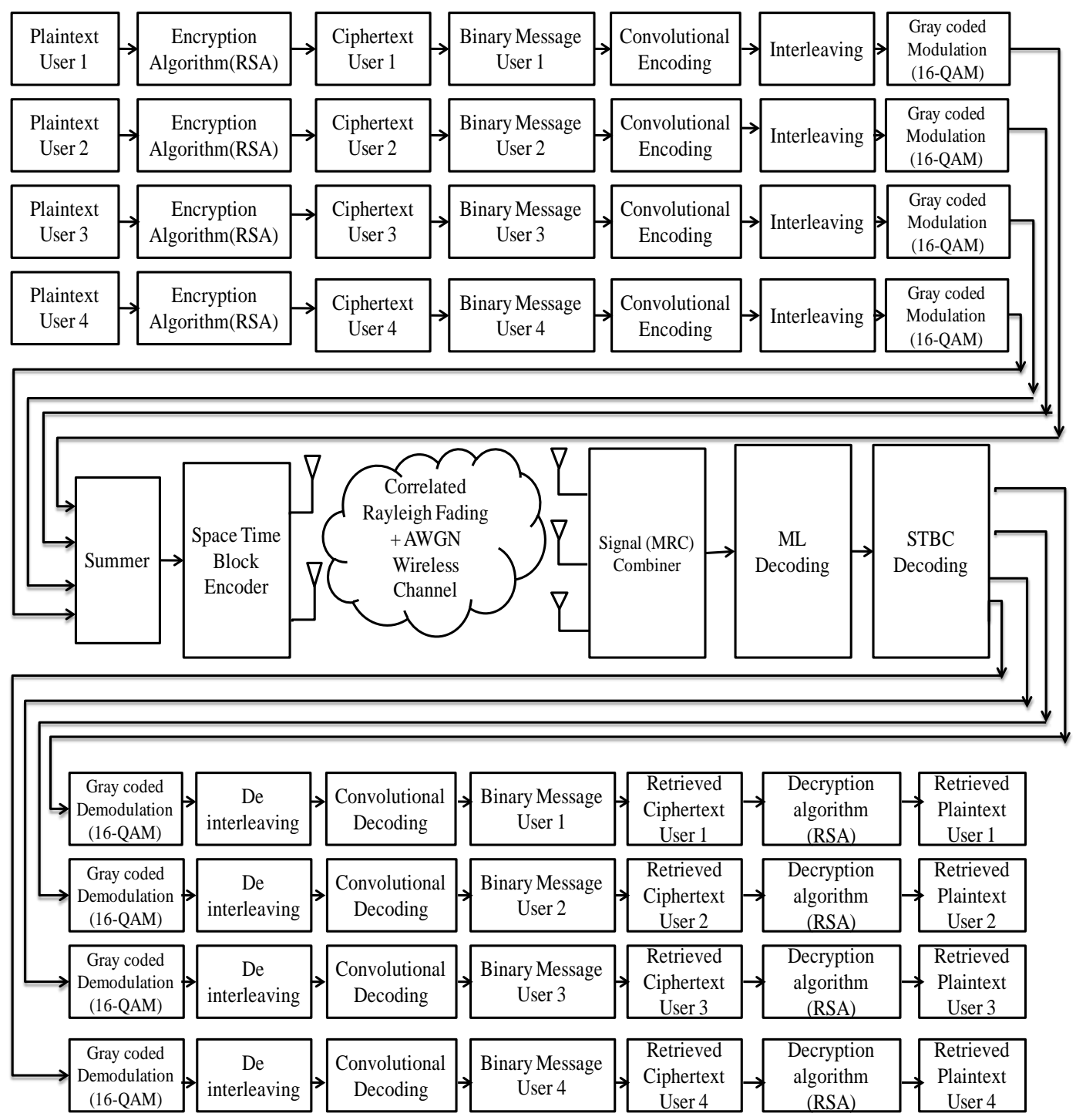

Figure 1. FEC Encoded MIMO STBC-MRC Secured Wireless Communication System

The maximum likelihood decoding rules for the two independent signals $x_{1}$ and $x_{2}$ are then

$$
\hat{x}_{1}=\arg \min _{\hat{x}_{1} \in S}\left[\left(\sum_{j=1}^{N_{R}}\left(\left|h_{j 1}\right|^{2}+\left|h_{j 2}\right|^{2}\right)-1\right)^{2}\left|\hat{x}_{1}\right|^{2}+d^{2}\left(\tilde{x}_{1}, \tilde{x}_{2}\right)\right]
$$




$$
\hat{x}_{2}=\arg \min _{\hat{x}_{2} \in S}\left[\left(\sum_{j=1}^{N_{R}}\left(\left|h_{j 1}\right|^{2}+\left|h_{j 2}\right|^{2}\right)-1\right)^{2}\left|\hat{x}_{2}\right|^{2}+d^{2}\left(\tilde{x}_{1}, \tilde{x}_{2}\right)\right]
$$

Where $d^{2}(x, y)=(x-y)\left(x^{*}-y^{*}\right)=|x-y|^{2}$

The complex symbols are now digitally demodulated, deinterleaved, convolutionally decoded and decrypted to recover the transmitted data for each of the four users.

\section{The Channel Model}

The correlated MIMO channel coefficients can now be generated by multiplying the uncorrelated MIMO fading channel vector by an $\mathrm{MN} \times \mathrm{MN}$ matrix $\mathrm{C}$, which is referred to as the following correlation-shaping matrix or symmetric mapping matrix [17], that is,

$$
\tilde{A}_{l}=\sqrt{P_{l}} C a_{l}
$$

where $P_{l}$ is the average power of the $l^{\text {th }}$ path, $a_{l}$ is a vector-form representation of the uncorrelated MIMO channel gain matrix $A_{l} . \tilde{A}_{l}$ is the correlated MN $\times 1$ MIMO channel vector.

The correlation-shaping matrix $\mathrm{C}$ defines the spatial correlation coefficients. A spatial correlation matrix is given as

$$
R=\left\{\begin{array}{c}
R_{B S} \otimes R_{M S}: \text { Downlink } \\
R_{M S} \otimes R_{B S}: \text { Uplink }
\end{array}\right.
$$

Where $\bigotimes$ denotes the Kronocker product. Using R in Equation (5) a root-power correlation matrix $\Gamma$ is given as

$$
\Gamma=\left\{\begin{aligned}
\sqrt{R}: & \text { For Filed Type } \\
R: & \text { For Complex Type }
\end{aligned}\right.
$$

Where $\Gamma$ is a non-singular matrix which can be decomposed into a symmetric mapping matrix (or correlation-shaping matrix) with the Cholesky or square-root decomposition as follows:

$$
\Gamma=\mathrm{CC}^{\mathrm{T}}
$$

$\mathrm{C}$ in Equation (7) can be obtained by Cholesky decomposition or square-root decomposition, depending on whether $R_{\mathrm{BS}}$ and $R_{\mathrm{MS}}$ are given as the complex matrices or real matrices, respectively.

\section{Performance Analysis and Result}

The computer simulation has been conducted to evaluate the BER performance of the FEC encoded multi user MIMO Alamouti's STBC-MRC secured wireless communication system based on the parameter given in Table 1.

To demonstrate the upshot of assorted multi-levels digital modulations (16-PSK and 16QAM) techniques over an Additive White Gaussian Noise (AWGN) and correlated Rayleigh Fading Channel first the effect of transmit diversity is investigated with no receive diversity in Figure 2 where the channel correlations varied from 0 to $90 \%$. 16-QAM modulation technique achieves better performance compared to the 16-PSK. At $14 \mathrm{~dB}$ SNR the BER performance improvements with the 16-QAM modulation technique are $7.32 \mathrm{~dB}, 7.50 \mathrm{~dB}$ and $7.78 \mathrm{~dB}$ respectively for $0 \%, 50 \%$ and $90 \%$ correlated channels. 
Table 1. Summary Table for the Simulated Model Parameters

\begin{tabular}{|c|c|}
\hline Type of input signal for the users & Secured Text Messages \\
\hline Public key for user 1 & $\{5,119\}$ \\
\hline Private key for user 1 & $\{77,119\}$ \\
\hline Public key for user 2 & $\{7,187\}$ \\
\hline Private key for user 2 & $\{23,187\}$ \\
\hline Public key for user 3 & $\{11,221\}$ \\
\hline Private key for user 3 & $\{35,221\}$ \\
\hline Public key for user 4 & $\{5,85\}$ \\
\hline Private key for user 4 & $2 \times$ N where N=1,2 and 3. \\
\hline No. of Transmitting and Receiving & \\
\hline antennas & $1 / 2$ rated Convolutional Encoding \\
\hline Channel coding & 16 -QAM and16-PSK \\
\hline Digital Modulation Techniques & AWGN and Rayleigh Fading channel \\
\hline Channel & 0 to 20 dBs \\
\hline Signal to noise ratio, SNR &
\end{tabular}

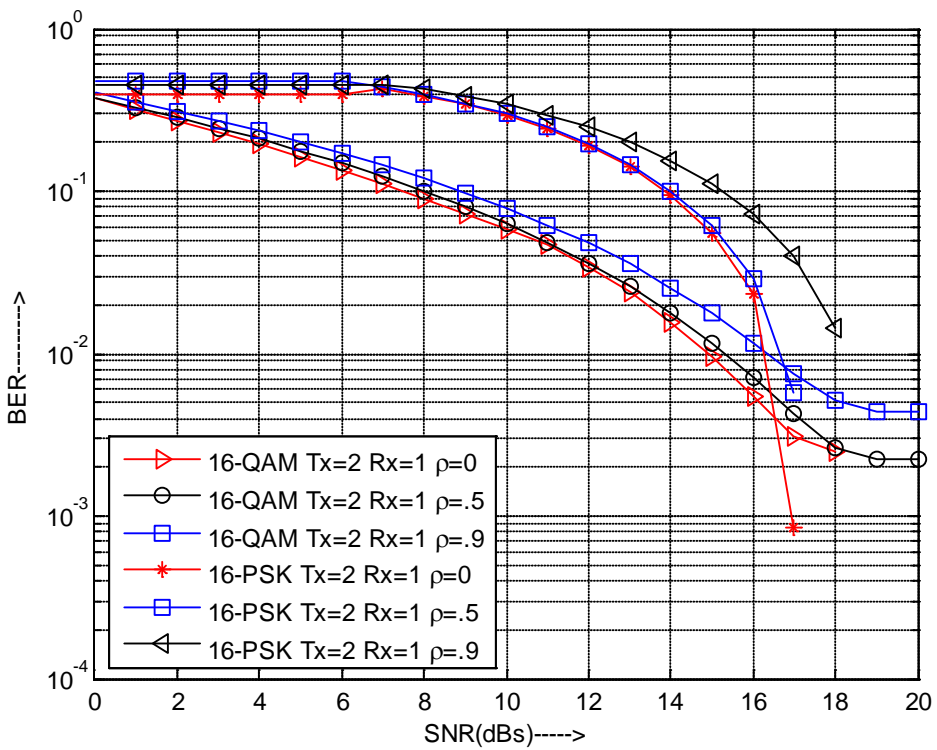

\section{Figure 2. BER Performance Analysis for 16-PSK and 16-QAM System with Transmitter and No Receiver Diversity}

Figure 3 and Figure 4 show the performance investigations when receiver diversity is considered with the transmit antenna diversity. In Figure 3 with the 16-QAM modulation technique the improved BER are recorded for $0 \%, 50 \%$ and $90 \%$ correlated channels respectively are $10.00 \mathrm{~dB}, 10.64 \mathrm{~dB}$ and $9.45 \mathrm{~dB}$. Again from Figure $4,2.85 \mathrm{~dB}, 4.35 \mathrm{~dB}$ and 6.52dB correspondingly BER improvements are observed for $0 \%, 50 \%$ and $90 \%$ correlated channels respectively by means of the 16-QAM method.

Finally in Figure 5 performance analyses are summarized intended for 16-QAM modulation technique in the company of $2 \times 2$ and $2 \times 3$ antenna arrangements. It is seen that for $0 \%, 50 \%$ and $90 \%$ correlated Rayleigh Fading channels respectively the BER 
performance enhancements are $4.70 \mathrm{~dB}, 5.85 \mathrm{~dB}$ and $1.38 \mathrm{~dB}$ respectively in case of $2 \times 3$ antennas compared to $2 \times 2$ antennas.

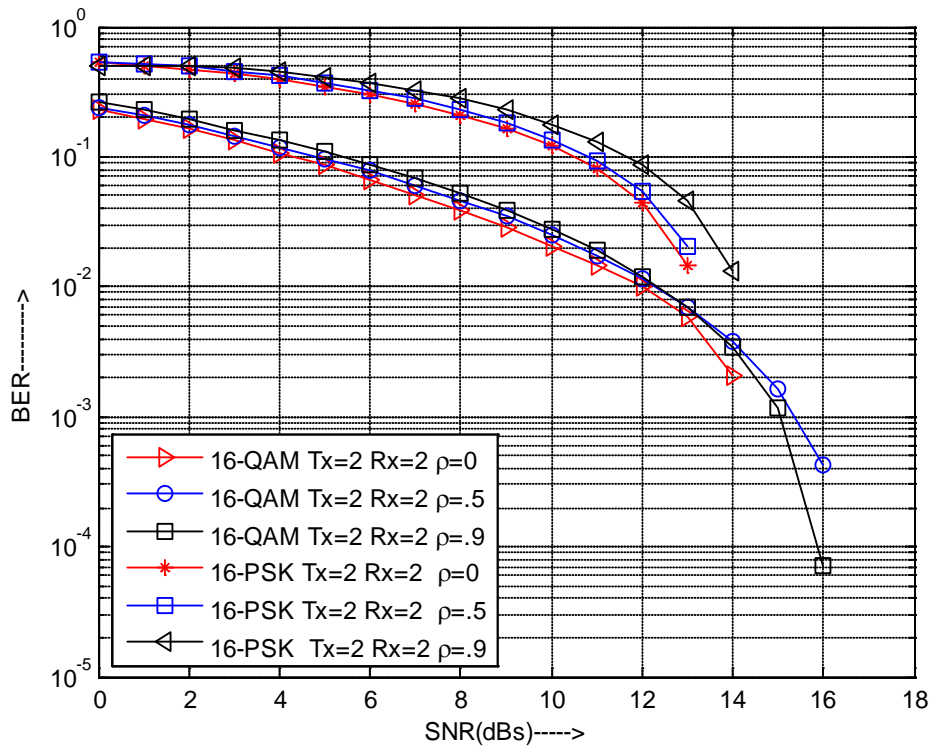

Figure 3. BER Performance Analysis for 16-PSK and 16-QAM System with Transmitter and Receiver Diversity ( $2 \times 2$ Antennas)

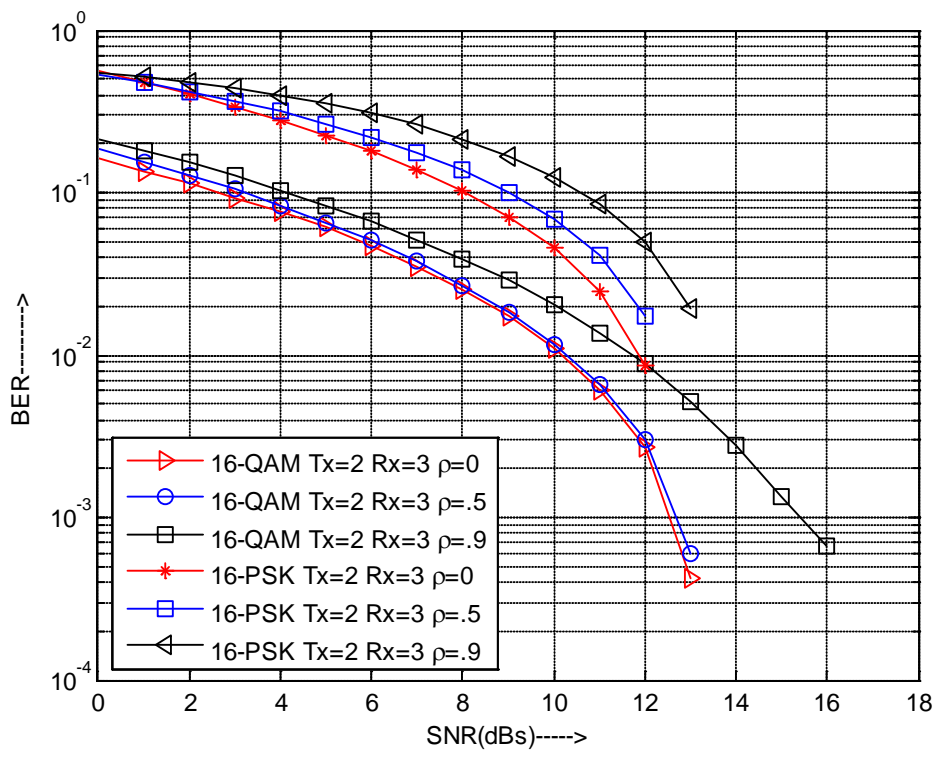

Figure 4. BER Performance Analysis for 16-PSK and 16-QAM System with Transmitter and Receiver Diversity ( $2 \times 3$ Antennas)

Under AWGN and correlated Rayleigh fading channel environment, the transmitted and received secured text messages are shown in Table 2 for four users at 17dB SNR for the FEC encoded Alamouti-MRC transmission system with $2 \times 1$ antennas and 16 PSK system. The erroneous characters in the retrieved text messages are shown in bold faces. 


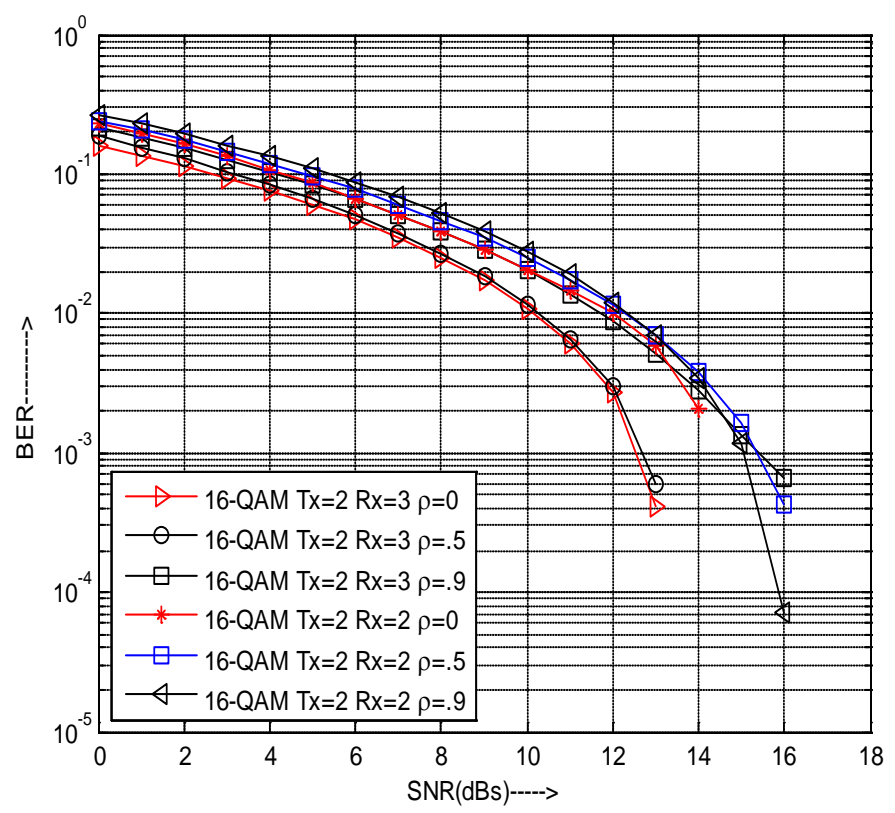

\section{Figure 5. BER Performance Analysis for16-QAM System with Transmitter and Receiver Diversity $(2 \times 2$ and $2 \times 3$ Antennas)}

Table 2. Transmitted and Received Secured Text Messages at 17 dB SNR

\begin{tabular}{|l|l|}
\hline \multicolumn{1}{|c|}{ Transmitted Plaintext } & \multicolumn{1}{c|}{ Retrieved Plaintext } \\
\hline $\begin{array}{l}\text { User1: Cryptography is the most important } \\
\text { aspect of communication systems security. }\end{array}$ & $\begin{array}{l}\text { User1: Cryptography is the most important aspect } \\
\text { OV communcation systems security. }\end{array}$ \\
\hline $\begin{array}{l}\text { User 2: Wireless communications is the fastest } \\
\text { growing segment of the communications } \\
\text { industry. }\end{array}$ & $\begin{array}{l}\text { User 2: Wirel commUnicatiLns :s the fastest } \\
\text { growing segment of the co'muni@atins industry. }\end{array}$ \\
\hline $\begin{array}{l}\text { User 3: Communication systems are designed } \\
\text { to transmit information bearing waveforms to } \\
\text { the receiver. }\end{array}$ & $\begin{array}{l}\text { User 3: Communication sys•ems are designed to } \\
\text { trasmit information bearing waveforms to } \\
\text { the8eceiver. }\end{array}$ \\
\hline $\begin{array}{l}\text { User 4: If there were no noise, messages can be } \\
\text { sent electronically to the outer limits of the } \\
\text { universe by using small amount of power. }\end{array}$ & $\begin{array}{l}\text { User 4: If there were no noise,messaOEs } \\
\text { sent electronicallDthe Uter limits of the univers } \\
\text { by using small amount 0f power. }\end{array}$ \\
\hline
\end{tabular}

\section{Conclusion}

In this work, we have presented simulation results concerning the adaptation of transmit and receive antenna diversity with digital multilevel modulation techniques in a multiuser MIMO Alamouti's STBC MRC secured wireless communication system. The system performance gets better with the more receiver diversity. In this context of system performance, it can be concluded that the implementation of 16-QAM digital modulation technique with $2 \times 3$ antenna arrangements provides acceptable result for such a correlated Rayleigh Faded multiuser Alamouti's STBC MRC secured wireless communication system. 


\section{References}

[1] E. Bigilieri, R. Calderbank, A. Constaninides, A. Goldsmith, A. Paulraj and H. Vincent Poor, "MIMO Wireless Communications”, Cambridge University Press, (2007).

[2] V. V. Veeravalli, "On Performance Analysis for Signaling on Correlated Fading Channels”, IEEE Transactions on Communications, vol. 49, no. 11, (2001) November, pp. 1879-1883.

[3] I. E. Telatar, "Capacity of multi-antenna Gaussian channels”, Eur. Trans. Commun., pp. 585-595, (1999) November-December.

[4] T. K. Y. Lo, “Maximum ratio transmission”, IEEE Trans. Commun., vol. 47, no. 10, pp. 1458-1461, (1999) October.

[5] M. R. McKay, Student Member, IEEE, A. J. Grant, Senior Member, IEEE and I. B. Collings , Senior Member, IEEE, "Performance Analysis of MIMO-MRC in Double-Correlated Rayleigh Environments", IEEE Transactions on Communications, vol. 55, no. 3, (2007) March, pp-497-507.

[6] K. Ashour Hamdi, Senior Member, IEEE, “Capacity of MRC on Correlate d Rician Fading Channels”, IEEE Transactions on Communications, vol. 56, no. 5, (2008) May, pp. 708-711.

[7] P. A. Dighe, R. K. Mallik and S. S. Jamuar, "Analysis of transmit-receive diversity in Rayleigh fading”, IEEE Trans. Commun., vol. 51, no.4, (2003) April, pp. 694-703.

[8] B. D. Rao and M. Yan, "Performance of maximal ratio transmission with two receive antennas", IEEE Trans. Commun., vol. 51, no. 6, (2003) June, pp. 894-895.

[9] M. Kang and M.-S. Alouini, "A comparative study on the performance of MIMO MRC with and without cochannel interference”, IEEE Trans. Commun., vol. 52, no. 8, (2004) August, pp. 1417-1425.

[10] A. J. Grant, "Performance analysis of transmit beamforming”, IEEE Trans. Commun., vol. 53, no. 4, (2005) April, pp. 738-744.

[11] Y. Chen and C. Tellambura, "Performance analysis of maximum ratio transmission with imperfect channel estimation”, IEEE Commun. Lett., vol. 9, no. 4, (2005) April, pp. 322-324.

[12] D. B. Smith and T. D. Abhayapala, "Maximal Ratio Combining Performance Analysis in Spatially Correlated Rayleigh Fading Channels with Imperfect Channel Knowledge”, Asia-Pacific Conference on Communications, (2005), pp. 549-553.

[13] L. Fang, G. Bi and A. C. Kot, "New method of performance analysis for diversity reception with correlated Rayleigh-fading signals”, IEEE Transactions on Vehicular Technology, vol. 49, no. 5, (2000), pp. 18071812.

[14] H. Zhu, Member, IEEE, B. Xia and Z. Tan, Member, IEEE, "Performance Analysis of Alamouti Transmit Diversity with QAM in Imperfect Channel Estimation”, IEEE Journal on Selected Areas in Communications, vol. 29, no. 6, (2011) June, pp. 1242-1248.

[15] H. Jafarkhan, "Space-Time Coding Theory and Practice”, (2005).

[16] F. Javier L'opez-Mart'ınez, E. Martos-Naya, K.-K. Wong and J. Tom'as Entrambasaguas, “Closed-Form BER Analysis of Alamouti-MRC Systems with ICSI in Ricean Fading Channels”, IEEE Communications Letters, vol. 15, no. 1, (2011) January, pp. 46-48.

[17] Y. Soo Cho, J. Kim, W. Young Yang and C. G. Kang, "MIMO-OFDM wireless communications with MATLAB”, (2010).

\section{Authors}

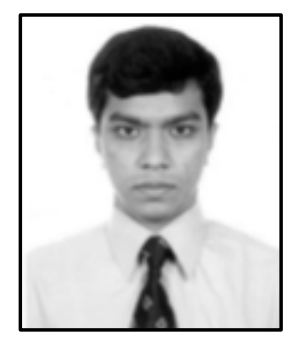

Tanvir Ahmed is an Assistant Professor of Electrical and Electronic Engineering (EEE) department, Faculty of Electrical \& Computer Engineering, Rajshahi University of Engineering and Technology (RUET), Rajshahi, Bangladesh. Before that he was a lecturer on the same department at RUET. He received his M.Sc. (Eng.) and B.Sc. (Eng.) degree in EEE from RUET in 2012 and 2009 respectively. His main research interests include MIMO-OFDM, MRC, STBC and Diversity techniques. He has several publications in International journal and conference papers. 


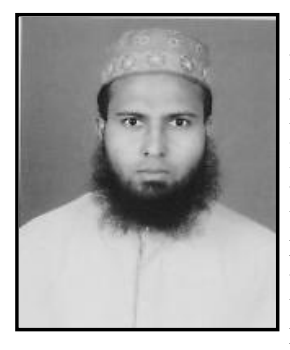

Md. Shamim Anower is an Assistant Professor of the Department of Electrical \& Electronic Engineering, Faculty of Electrical \& Computer Engineering, Rajshahi University of Engineering \& Technology, Bangladesh. He received his BSc and MSc both in Electrical \& Electronic Engineering from the same university in 2002 and 2007 respectively. Moreover he received his $\mathrm{PhD}$ degree in Electrical Engineering from UNSW, Canberra, Australia in 2012. His main research interests include Wireless communications technology, Underwater wireless communication, Signal processing applications, Power line communication. He has published more than 40 articles in Electrical and Electronic Engineering field.

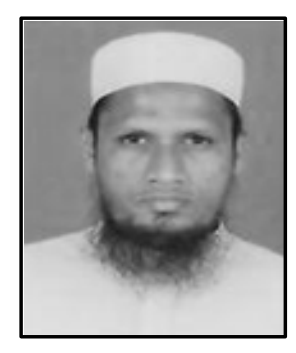

Md. Zahurul Islam Sarkar is an Assistant Professor of the Department of Electrical and Electronic Engineering, Faculty of Electrical \& Computer Engineering, Rajshahi University of Engineering \& Technology, Bangladesh. He obtained his M. Sc. Engg. and B. Sc. Engg. degrees both in Electrical and Electronic Engineering from Rajshahi University of Engineering \& Technology, Bangladesh, in 2000 and 1996, respectively. He received his $\mathrm{PhD}$ degree in Electrical and Electronic Engineering from Queen's University Belfast, United Kingdom in February 2012. From September 2007 to December 2008, Mr. Sarkar worked as a Research Assistant at the Communications and Coding Theory Laboratory (CCTLAB), Kyung Hee University, South Korea. He received the Best Paper Award at the $67^{\text {th }}$ IEEE Vehicular Technology Conference (VTC2008-Spring). His main research interests center around the Information-Theoretic Security in Wireless Communication Systems, Secure Wireless Multicasting, Random Coding Error Exponent for MIMO Channels, Interference Alignment, Precoding Techniques, Digital Signal Processing, Adaptive Signal Processing, Cognitive Radio, Cooperative Communications and Analysis of Lightning Induced Electromagnetic Fields. He has published more than 50 articles in the field of Electrical and Electronic Engineering.

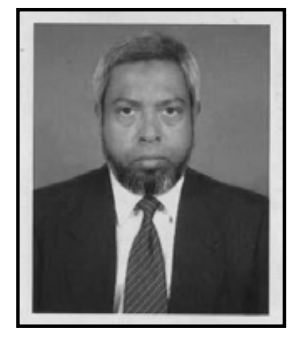

Md. Mortuza Ali is a Professor of the Department of Electrical \& Electronic Engineering, Faculty of Electrical \& Computer Engineering, Rajshahi University of Engineering \& Technology, Bangladesh. He received his B.Sc (Eng.) in Electrical \& Electronic Engineering from University of Rajshahi in 1979, and M.S and Ph. D degree both from Niigata University, Japan in 1989 and 1992, respectively. His main research interests include High Power Microwave Devices, MIMO-OFDM, WiMAX, Cognitive radio and LTE radio interface technologies. 
International Journal of Advanced Science and Technology Vol.58, (2013) 